\title{
miR-483-5p plays a protective role in chronic obstructive pulmonary disease
}

\author{
ZHENYU SHEN $^{1}$, WENXIANG TANG $^{2}$, JIANG GUO $^{3}$ and SHENGHUA SUN ${ }^{2}$ \\ ${ }^{1}$ Department of Respiratory Medicine, Xiangtan Central Hospital, Xiangtan, Hunan 411100; \\ ${ }^{2}$ Deparment of Respiratory Medicine, The Third Xiangya Hospital of Central South University, Changsha, \\ Hunan 410013; ${ }^{3}$ Cardio-Thoracic Surgery, Xiangtan Central Hospital, Xiangtan, Hunan 411100, P.R. China
}

Received June 15, 2016; Accepted May 8, 2017

DOI: $10.3892 /$ ijmm.2017.2996

\begin{abstract}
Altered microRNA (miRNA or miR) expression has been reported in chronic obstructive pulmonary disease (COPD). The present study aimed to identify the involvement of miRNAs in the pathophysiology of COPD and to explore the effects of various miRNAs with significant alteration on COPD in vitro. We conducted high-throughput analysis of miRNAs (miRNA microarray) in lung samples from 10 COPD patients and 10 healthy persons with a validation experiment using quantitative (real-time) polymerase chain reaction (real-time PCR) panels. By analyzing 3,000 miRNAs in lung samples using a microarray, we identified 341 differentially expressed miRNAs (138 with high expression and 203 with low expression) in patients with COPD in comparison with the healthy controls. Then 15 high-expression candidates and 15 low-expression candidates with at least 2 -fold difference and $\mathrm{P}<0.05$ were selected randomly to validate the changes in three independent experiments in vitro using real-time PCR. The validation test showed a positive correlation with the microarray results. Then we chose miR-483-5p as our target. The effect of miR-483-5p on cell proliferation and expression of COPD-related proteins were detected using Cell Counting Kit 8 and western blot analysis, respectively. The results showed that miR-483-5p, which was significantly downregulated in COPD samples, abrogated the transforming growth factor- $\beta$ (TGF- $\beta$ )-mediated decrease in cell proliferation, and increase in $\alpha$-smooth muscle actin ( $\alpha$-SMA) and fibronectin expression in pulmonary epithelial and lung fibroblast cell lines, BEAS-2B and HFL1. These findings suggest that miR-483-5p may play an important and protective role in patients with COPD and may serve as a
\end{abstract}

Correspondence to: Dr Shenghua Sun, Department of Respiratory Medicine, The Third Xiangya Hospital of Central South University, 3w 138 Tongzipo Road, Yuelu, Changsha, Hunan 410013, P.R. China E-mail:ssh88_doc@sina.com

Key words: chronic obstructive pulmonary disease, miRNA, microarray, miR-483-5p useful biomarker and for early detection of COPD as well as a potential therapeutic tool.

\section{Introduction}

microRNAs (miRNAs or miRs) are a family of small noncoding RNAs which modulate gene expression by binding to complementary sequences of target mRNAs in the coding or non-coding region such as the 3 ' untranslated region (3'UTR) and 5'UTR (1). The mature miRNAs cause post transcriptional gene repression by increasing mRNA degradation or by inhibiting translation (2). In the human body, miRNAs play important roles in the responses to injury or adaptation to chronic stress. More and more studies have revealed that specific miRNAs can serve as biomarkers during disease progression and development, such as disorders of the lung, by regulating cell proliferation and differentiation (3-6).

Chronic obstructive pulmonary disease (COPD) is considered as a type of airway disorder and respiratory disease, which is associated with persistent inflammation (7). It may become the third leading cause of death by 2020 globally (8). Usually this type of chronic condition is influenced by a combination of environmental, genetic and epigenetic components and physiological changes. Different signaling pathways and important molecular biomarkers involved in the progression of chronic inflammation in lung disorders have been presented in miRNA studies $(9,10)$. miRNAs, such as miR-218 and miR-128b, are regulators of smoking-induced gene expression alterations in human airway epithelium (11). Scientists have also found that cigarette smoke condensate increases the expression of miR-31 in airway cells (12), and let-7d is involved in idiopathic pulmonary fibrosis (13). Recently the expression of let-7c and miR-125b in sputum samples from patients with COPD was demonstrated to be much lower than levels in healthy controls (14). In lung tissue samples from patients with COPD, high expression of miR-199a-5p and miR-34a is associated with downregulation of $\mathrm{HIF}-1 \alpha$ protein expression which is important during the progression of COPD (15). We believe that numerous functional miRNAs associated with COPD are still unknown.

Thus, a completed profile of alternative miRNAs in lung samples from COPD patients and healthy donors was detected by microarray analysis in this study. According to the results, 
15 miRNAs with high expression and 15 with low expression were selected and validated in vitro using real-time PCR. Finally, miR-483-5p was selected as a study candidate and, importantly, miR-483-5p transfection significantly inhibited the transforming growth factor- $\beta$ (TGF- $\beta$ )-mediated decrease in cell proliferation, and $\alpha$-smooth muscle actin ( $\alpha$-SMA) and fibronectin expression in BEAS-2B and HFL1 cells in vitro. Our results suggest that miR-483-5p plays an important and protective role in patients with COPD.

\section{Materials and methods}

Patient characteristics, clinical features and serum harvest. This study was approved by the Third Xiangya Hospital, Central South University (Hunan, China); and an informed consent form (ICF) was provided by each participant. All of the patients gave informed consent to have their tissues banked.

Lung tissue samples from 20 patients were included in this study and were divided into two groups according to the Global Initiative for Chronic Obstructive Lung Disease (GOLD) classification. Ten samples were collected from patients with normal lung function (no COPD, $n=10$ ); the rest of the samples were from patients with diagnosed COPD ( $n=10$; stage I/II/III, GOLD classification). Table I summarizes the patient characteristics and clinical features. The diagnosis of emphysema was made by a pathologist based on histological examination, and all of the lung tissue samples collected from patients with COPD had centrilobular emphysema.

All lung tissue samples were maintained at $-80^{\circ} \mathrm{C}$ until the processing of total RNA isolation.

Chemicals. TGF- $\beta$ was purchased from Sigma (St. Louis, MO, USA). Other chemicals were commercially available and purchased as reagent grade from Sinopharm (Shanghai, China).

Cell culture and treatment. Human normal pulmonary epithelial BEAS-2B cells obtained from the American Type Culture Collection (ATCC, Manassas, VA, USA) were cultured in growth media containing Roswell Park Memorial Institute-1640 (RMPI-1640) medium, supplemented with no serum and $1 \%$ penicillin-streptomycin (Mediatech, Herndon, VA, USA) at $37^{\circ} \mathrm{C}$ in a humidified atmosphere of $5 \% \mathrm{CO}_{2}$ in air. Within the same culture condition, human normal lung fibroblast HFL1 cells (ATCC) were cultured in growth media containing Ham's F12K medium (F12K).

Before being diluted into single-cell suspensions and seeded in 12 -well plates $\left(1 \times 10^{5}\right.$ cells $\left./ \mathrm{ml}\right)$, the cells were treated with or without TGF- $\beta$ ( $1 \mathrm{mg} / \mathrm{ml})$ for $24 \mathrm{~h}$, and then transfected with or without different miRNA mimics. Finally, the cells were harvested for total protein isolation. The cells receiving no treatment served as the negative control group, and cells with TGF- $\beta$ only treatment served as the positive control group.

Total RNA isolation and reverse transcription. Total RNA from the lung sample short RNAs $(<200 \mathrm{bp})$ was harvested and extracted using an RNA Mini Elute kit (Qiagen, Venlo, The Netherlands) according to the manufacturer's instructions. RNA quality was ascertained using Agilent 2100 bioanalyzer (Agilent Technologies, Santa Clara, CA, USA). One microgram of total RNA was reverse-transcribed and the product $(11 \mu \mathrm{l})$ was pre-amplified using Megaplex PreAmp Primers and DBI Bestar $^{\circledR}$ qPCR RT kit (Applied Biosystems, Foster City, CA, USA) in a $20-\mu 1$ PCR reaction. The pre-amplification cycling conditions were $37^{\circ} \mathrm{C}$ for $60 \mathrm{~min}$ and $98^{\circ} \mathrm{C}$ for $10 \mathrm{~min}$. The pre-amplified cDNA was diluted with $0.1 \mathrm{X} \mathrm{TE}(\mathrm{pH} \mathrm{8.0)}$ to $10 \mu \mathrm{l}$ and then $1 \mu \mathrm{l}$ diluted cDNA was used in each plate for real-time PCR reactions.

miRNA microarray labeling and hybridization. Extracted RNA was quantitated using a NanoDrop ND-1000 spectrophotometer (NanoDrop, Wilmington, DE, USA) and monitored by agarose gel electrophoresis. Then, the samples were labeled and hybridized on Affymetrix GeneChip miRNA arrays 3.0 (Affymetrix, Santa Clara, CA, USA) according to the manufacturer's protocol. Samples were denatured at $99^{\circ} \mathrm{C}$ for $5 \mathrm{~min}$ followed by $45^{\circ} \mathrm{C}$ for another $5 \mathrm{~min}$, injected into the array chips and hybridization was allowed for $17 \mathrm{~h}$ at $48^{\circ} \mathrm{C}$ in an Affymetrix Hybridization Oven 645 in constant movement at $60 \mathrm{rpm}$. The raw intensity of the image was read using GenePix Pro V6.0. The intensity of the green signal was calculated after background subtraction, and four replicated spots for each probe on the same slide were averaged. The median normalization method was used to obtain 'normalized data' $[$ Normalized data $=($ foreground - background $) /$ median $]$. The median was defined as the $50 \%$ quantile of miRNA intensity that was $>50$ in all samples after background correction. The statistical significance of the differentially expressed miRNAs was analyzed using the Student's t-test.

Quantitative RT-PCR of mature miRNAs. The solution contained $1 \mu \mathrm{l}$ of RT product, $5 \mu \mathrm{l}$ of $2 \mathrm{X} \mathrm{SYBR}{ }^{\circledR}$-Green Mix, $0.5 \mu \mathrm{l}$ of each primer and $3 \mu \mathrm{l}$ nuclease-free water. The reactions were performed in a 96 -well optical plate at $94^{\circ} \mathrm{C}$ for $2 \mathrm{~min}$, followed by 40 cycles of $94^{\circ} \mathrm{C}$ for $20 \mathrm{sec}, 58^{\circ} \mathrm{C}$ for $20 \mathrm{sec}$ and $72^{\circ} \mathrm{C}$ for $20 \mathrm{sec}$, and the fluorescence signal was collected by ABI PRISM ${ }^{\circledR} 7900$ HT system (Applied Biosystems). All reactions were run in triplicate. All primers used are listed in Table II.

Cell proliferation detection. After TGF- $\beta$ treatment for $24 \mathrm{~h}$, miR-483-5p mimics and miRNA mimic negative control (NC) (1 $\mu \mathrm{g}$; GenePharma, Shanghai, China) were transfected into BEAS-2B and HFL1 cells using Lipofectamine ${ }^{\mathrm{TM}} 2000$ (Invitrogen, Carlsbad, CA, USA). Then, after transfection for 24, 48 and 72 h, $100 \mu$ l Cell Counting Kit-8 (CCK-8) (Dojindo, Kumamoto, Japan) solution was added into each well and the plates were incubated in a incubator for $1 \mathrm{~h}$. The absorbance was measured at a wavelength of $450 \mathrm{~nm}$ using a microplate reader.

Protein isolation and western blot analysis. To evaluate the target gene expression change in vitro affected by miR-483-5p, the protein extracted from the cells was lysed using RIPA buffer $(150 \mathrm{mM} \mathrm{NaCl}, 1 \%$ Nonidet P-40, 0.1\% sodium dodecyl sulfate (SDS), $50 \mathrm{mM}$ Tris- $\mathrm{HCl} \mathrm{pH}$ 7.4, $1 \mathrm{mM}$ EDTA, $1 \mathrm{mM}$ PMSF, 1X Roche complete mini protease inhibitor cocktail, Roche PhosSTOP phosphatase inhibitor cocktail) and then determined using BCA kit (Pierce, Rockford, IL, USA) and $20 \mu \mathrm{g}$ protein lysates were separated on $10 \%$ SDS-PAGE gels followed by transfer to nitrocellulose membranes. Western 
Table I. Demographic, clinical and biological data of the COPD patients and healthy controls in the miRNA screen study.

\begin{tabular}{lcc}
\hline & $\begin{array}{c}\text { COPD } \\
(\mathrm{N}=10)\end{array}$ & $\begin{array}{c}\text { Healthy controls } \\
(\mathrm{N}=10)\end{array}$ \\
\hline Gender (male), $\mathrm{n}(\%)$ & $8(80)$ & $1(10)$ \\
Age (years) & $70.43( \pm 16.25)$ & $60.25( \pm 15.89)$ \\
FEV1/FVC, $\%$ & $55.70( \pm 7.28)$ & $81.35( \pm 5.92)$ \\
FEV1, \% predicted & $59.76( \pm 9.95)$ & $92.35( \pm 5.14)$ \\
BDR, \% & $6.30( \pm 1.90)$ & $2.35( \pm 1.92)$ \\
Smoking, pack-years & $41.00( \pm 32.09)$ & 0 \\
Current smoker, $\mathrm{n}$ & 0 & 0 \\
Medication, $\mathrm{n}$ & & 0 \\
Oral corticosteroid & 1 & 0 \\
Inhaled corticosteroid & 0 & 2 \\
Lung cancer diagnosis, $\mathrm{n}$ & 9 & \\
\hline
\end{tabular}

Values are expressed as means $( \pm \mathrm{SD})$. COPD, chronic obstructive pulmonary disease; BDR, bronchodilator response.

blot analysis was performed as previously described (16), and the signal was visualized using the Odyssey Imaging system (LI-COR Bioscience, Lincoln, NE, USA). Antibodies used in this study included anti-human $\alpha$-SMA $(1: 4,000)$, anti-human fibronectin $(1: 2,000)$ and anti-human glyceraldehyde 3-phosphate dehydrogenase (GAPDH) (1:10,000) (all from Santa Cruz Biotechnology, Inc., Santa Cruz, CA, USA).

Data analysis. The miRNA microarray data used the total gene signal, which was proportional to the total number of targets bound by the probes targeting each miRNA. Differentially expressed signals were determined by one-way ANOVA with $\mathrm{P}<0.01$. To compare qPCR-array and microarray assays, the $\log _{2}$ of microarray signals was used.

Real-time PCR assay was used to determine the changes in the expression of the target miRNAs in cells or lung tissue samples. The change in amplification was normalized to the expression of U6 RNA. The fold-change in expression was calculated for each sample using $2^{-\Delta \Delta \mathrm{Ct}} .2^{-\Delta \Delta \mathrm{Ct}}>1.5$ or $<0.67$ was indicative of miRNAs that were differentially expressed.

\section{Results}

Differentially expressed miRNAs in COPD patients. A total of 3,000 miRNAs were identified. Among these, 138 miRNAs were differentially expressed with a $>2$-fold-change in the lung tissues between COPD patients and healthy donors, and 203 miRNAs had significantly downregulated expression (Fig. 1).

Validation of miRNA microarray results in clinical samples by real-time $P C R$. In order to confirm the results obtained from the miRNA microarray, 15 high-expression candidates and 15 low-expression candidates with at least a 2-fold difference and $\mathrm{P}<0.05$ were randomly selected from the result identified by the microarray, and the expression of these miRNAs was analyzed by real-time PCR. As shown in Fig. 2A, hsa-miR-24-3p,
Table II. Sequence of the primers used for validation of selected miRNAs.

\begin{tabular}{ll}
\hline miRNA & \multicolumn{1}{c}{ Primers (5'-3') } \\
\hline hsa-miR-24-3p & F: ACACTCCAGCTGGGTGGCTCAGTTCAGC \\
hsa-miR-101-3p & F: ACACTCCAGCTGGGTACAGTACTGTGAT \\
hsa-miR-125a-5p & F: ACACTCCAGCTGGGTCCTGAGACCCTTTA \\
hsa-miR-30c-5p & F: ACACTCCAGCTGGGTGTAAACATCCTACAC \\
hsa-let-7b-5p & F: ACACTCCAGCTGGGTGAGGTAGTAGGTTG \\
hsa-miR-193a-3p & F: ACACTCCAGCTGGGAACTGGCCTACAAAG \\
hsa-miR-200c-3p & F: ACACTCCAGCTGGGTTAATACTGCCGGGTA \\
hsa-miR-140-3 & F: ACACTCCAGCTGGGTACCACAGGGTAGAAC \\
hsa-miR-22-3p & F: ACACTCCAGCTGGAAGCTGCCAGTTGAAG \\
hsa-miR-195-5p & F: ACACTCCAGCTGGGTAGCAGCACAGAAAT \\
hsa-miR-4328 & F: ACACTCCAGCTGGGCCAGTTTCCCAG \\
hsa-miR-16-5p & F: ACACTCCAGCTGGGTAGCAGCACGTAAA \\
hsa-miR-141-3p & F: ACACTCCAGCTGGGTAACACTGTCTGGTAA \\
hsa-miR-146b-5p & F: ACACTCCAGCTGGGTGAGAACTGAATTCC \\
hsa-miR-191-5p & F: ACACTCCAGCTGGGCAACGGAATCCCAAAAG \\
hsa-miR-4451 & F: ACACTCCAGCTGGGTGAGAACTGAATTCC \\
hsa-miR-204-3p & F: ACACTCCAGCTGGGGCTGGGAAGGCA \\
hsa-miR-340-5p & F: ACACTCCAGCTGGGTTATAAAGCAATGAG \\
hsa-miR-3611 & F: ACACTCCAGCTGGGTTGTGAAGAAAGAA \\
hsa-miR-665 & F: ACACTCCAGCTGGGACCAGGAGGCTGAGG \\
hsa-miR-483-5p & F: ACACTCCAGCTGGGAAGACGGGAGGAA \\
hsa-miR-4644 & F: ACACTCCAGCTGGGTGGAGAGAGAAAAGAG \\
hsa-miR-485-3p & F: ACACTCCAGCTGGGGTCATACACGGCTCTC \\
hsa-miR-4698 & F: ACACTCCAGCTGGGTCAAAATGTAGAGG \\
hsa-miR-185-5p & F: ACACTCCAGCTGGGTGGAGAGAAAGGCAG \\
hsa-miR-659-3p & F: ACACTCCAGCTGGGCTTGGTTCAGGGAGGG \\
hsa-miR-378a-3p & F: ACACTCCAGCTGGGACTGGACTTGGAG \\
hsa-miR-3653-3p & F: ACACTCCAGCTGGGCTAAGAAGTTGAC \\
hsa-miR-4421 & F: ACACTCCAGCTGGGACCTGTCTGTGGAAAG \\
hsa-miR-663a & F: ACACTCCAGCTGGGAGGCGGGGCGCCGCG \\
U6 & F: CTCGCTTCGGCAGCACA \\
U6 & R: AACGCTTCACGAATTTGCGT \\
All & \\
&
\end{tabular}

F, forward; R, reverse.

hsa-miR-101-3p, hsa-miR-125a-5p, hsa-miR-30c-5p, hsa-let-7b-5p, hsa-miR-146-5p,hsa-miR-193a-3p, hsa-miR-200c-3p, hsa-miR-140-3p, hsa-miR-22-3p, hsa-miR-195-5p, hsa-miR-16-5p, hsa-miR-141-3p, hsa-miR-4328 and hsa-miR-191-5p were upregulated and hsa-miR-4451, hsa-miR-204-3p, hsa-miR-340-5p, hsa-miR-3611, hsa-miR-665, hsa-miR-483-5p, hsa-miR-4644, hsa-miR-485-3p, hsa-miR-4698, hsa-miR-185-5p, hsa-miR-659-3p, hsa-miR-378a-3p, hsa-miR-3653-3p, hsa-miR-4421, and hsa-miR-663a were downregulated in each lung sample (Fig. 2A), which was consistent with the results from the miRNA microarray (Fig. 2B and C). Among these candidates, we chose miR-483-5p as our target for the following detections.

Effect of miR-483-5p on cell proliferation in vitro. Furthermore, we examined the effects of miR-483-5p on TGF- $\beta$-treated BEAS-2B and HFL1 cell proliferation after transfection for 24, 48 and 72 h. As shown in Fig. 3A, miR-483-5p, which was significantly downregulated in COPD samples, exhibited an inhibitory effect against the TGF- $\beta$-induced decrease in cell proliferation compared to the negative control group in the BEAS-2B and HFL1 cells (Fig. 3B). 


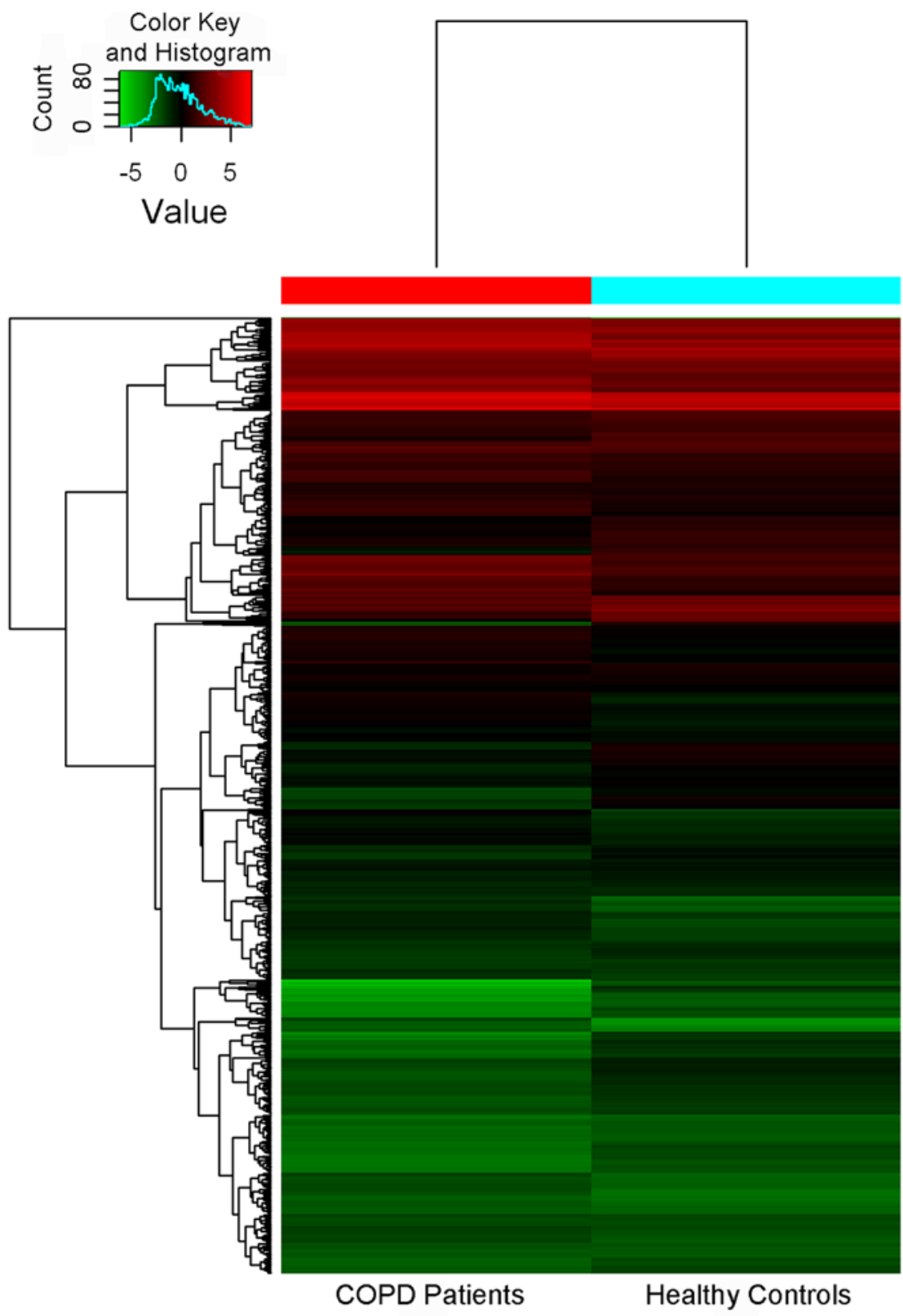

Figure 1. Heat map of the differentially expressed microRNAs (miRNAs) in fracture healing. Red represents upregulation and green represents downregulation, black represents no differential expresssion. Color shade represents the intensity of fluorescence and reflects the level of hsa-miRNA expression. The scheme indicates that the clustering properties of gene expression in chronic obstructive pulmonary disease (COPD) patients were obvious.

Effect of miR-483-5p on expression of COPD-related proteins. In order to explore the effect of miR-483-5p in COPD, we harvested BEAS-2B and HFL1 cells after TGF- $\beta$ treatment for $24 \mathrm{~h}$ followed by miR-483-5p mimic transfection. As a result, we found that the protein levels of $\alpha$-SMA and fibronectin were decreased $48 \mathrm{~h}$ post-transfection (Fig. 4).

\section{Discussion}

COPD is a debilitating lung disease that generally affects older individuals, owing to the duration of smoking (17). miRNAs may be quiescent while lung homeostasis is maintained after development, but may become perturbed in early states of COPD involving cell differentiation and inflammation (18). In this study, we report that COPD, rather than smoking, has a significant impact on the miRNA expression profile based on
miRNA microarray analysis using lung samples from patients with and without COPD. Consistent with the microarray results, expression levels of certain miRNAs were validated in vitro by real-time PCR.

Recently, miR-483-3p has been reported to be involved in the occurrence of many diseases, such as esophageal squamous cell carcinoma (19), cardiomyocyte apoptosis (20) and gastric cancer (21). It has also been shown to be dysregulated and associated with poorer disease-specific survival in various cancers (22-26). Although Song et al (24) reported that miR-483-5p can serve as a negative regulator of lung cancer metastasis suppressors RhoGDI1 and ALCAM, the role of miR-483 in lung disease particularly COPD and the molecular mechanisms by which miR-483 regulates such diseases are still not clear. Meanwhile, Soeda et al (27) also found that miR-483-5p was significantly downregulated in the plasma 


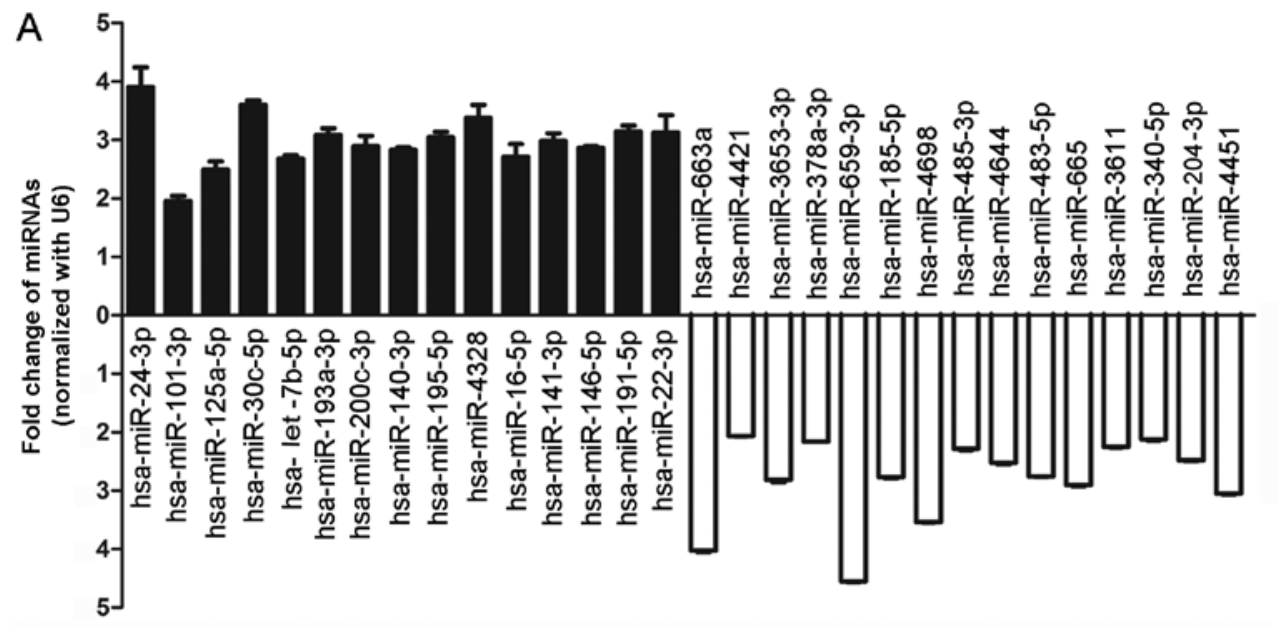

B
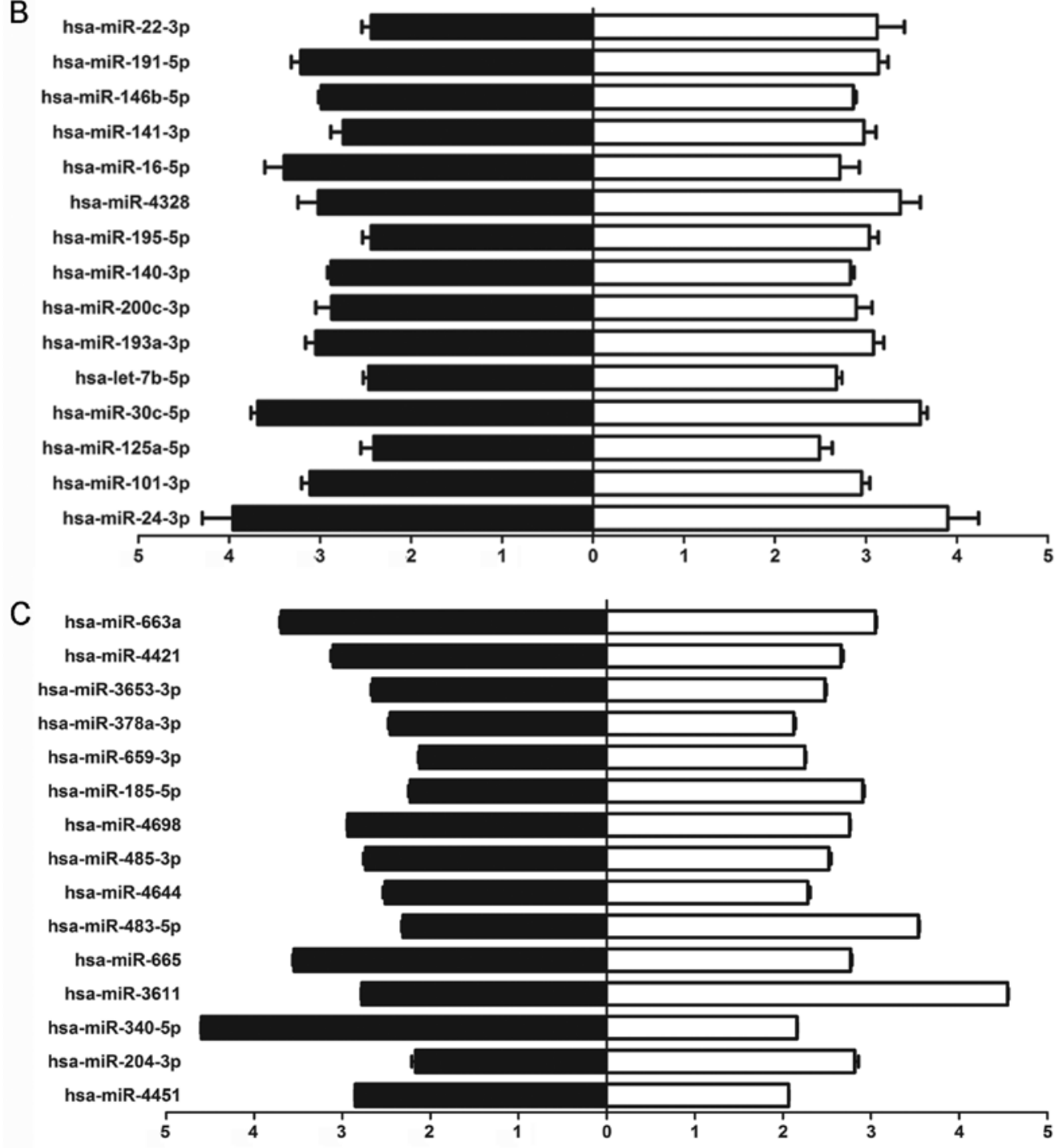

Figure 2. Thirty differentially expressed hsa-miRNAs related to disease sensitivity between cohort chronic obstructive pulmonary disease (COPD) patients and healthy controls were screened and identified by real-time PCR. (A) Validation of 30 hsa-miRNAs using real-time PCR showed that hsa-miR-24-3p, hsa-miR-101-3p, hsa-miR-125a-5p, hsa-miR-30c-5p, hsa-miR-30d-5p, hsa-let-7b-5p, hsa-miR-193a-3p, hsa-miR-200c-3p, hsa-miR-140-3p, hsa-miR-22-3p, hsa-miR-195-5p, hsa-miR-16-5p, hsa-miR-141-3p, hsa-miR-30b-5p and hsa-miR-191-5p were upregulated and hsa-miR-4451, hsa-miR-204-3p, hsa-miR-3611, hsa-miR-665, hsa-miR-483-5p, hsa-miR-4644, hsa-miR-485-3p, has-miR-185-5p, hsa-miR-4698, hsa-miR-185-5p, hsa-miR-659-3p, hsa-miR-378a-3p, hsa-miR-3653-3p, hsa-miR-4421 and hsa-miR-663a were downregulated in each lung sample from COPD patients. Black, relative change in COPD patients normalized with the control group; white, relative change in healthy donors normalized to COPD patients. (B) High level of 15 hsa-miRNAs randomly selected for the validation of expression level in COPD patients by real-time RT-PCR was consistent with the results from the miRNA microarray. Black, relative change in COPD patients normalized with the control group as detected by real-time PCR; white, relative change in COPD patients normalized to the control group as detected by microarray. (C) Low level of 15 hsa-miRNAs randomly selected for the validation of expression level in COPD patients by real-time RT-PCR was consistent with the results from miRNA microarray. Black, relative change in healthy donors normalized to COPD patients as detected by real-time PCR; white, relative change in healthy donors normalized to the COPD patients as detected by the microarray. 

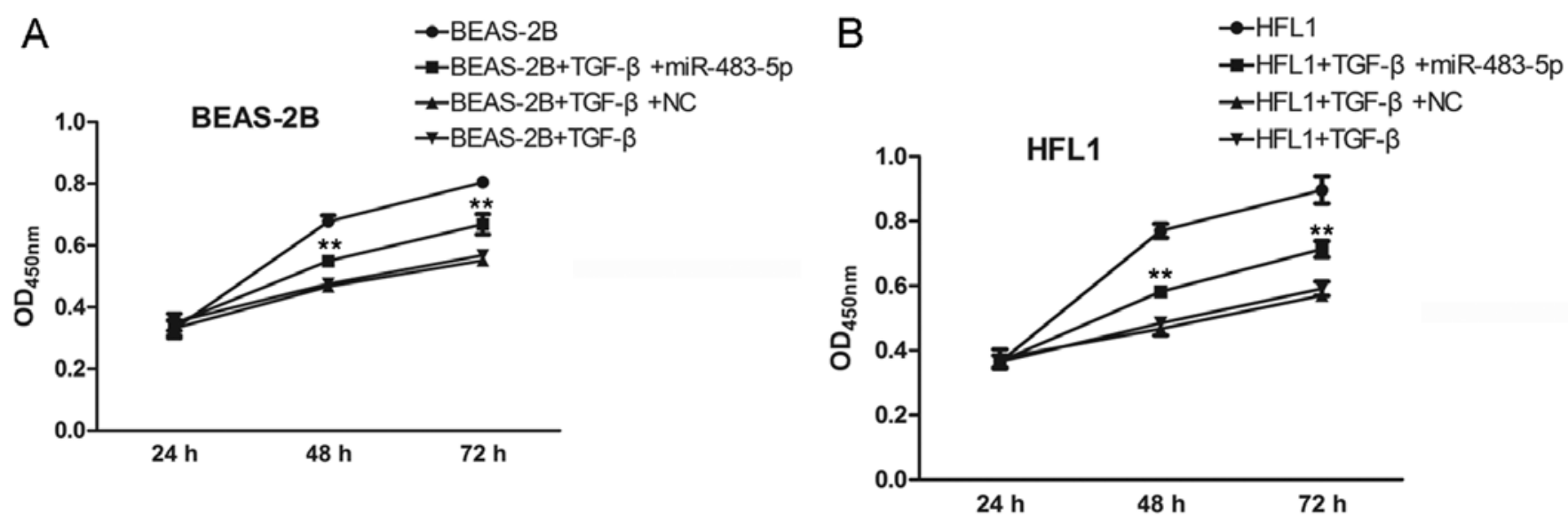

Figure 3. Upregulation of miR-483-5p promotes the proliferation of (A) BEAS-2B and (B) HFL1 cells in vitro. Cell numbers were counted at the following time-points: 24,48 and $72 \mathrm{~h}$. Cell viability was measured using the CCK-8 assay. Data are shown as the mean \pm standard deviation. All experiments were repeated independently for three times. ${ }^{* *} \mathrm{P}<0.01 \mathrm{vs}$. the group with transforming growth factor- $\beta(\mathrm{TGF}-\beta)$ treatment only.

A

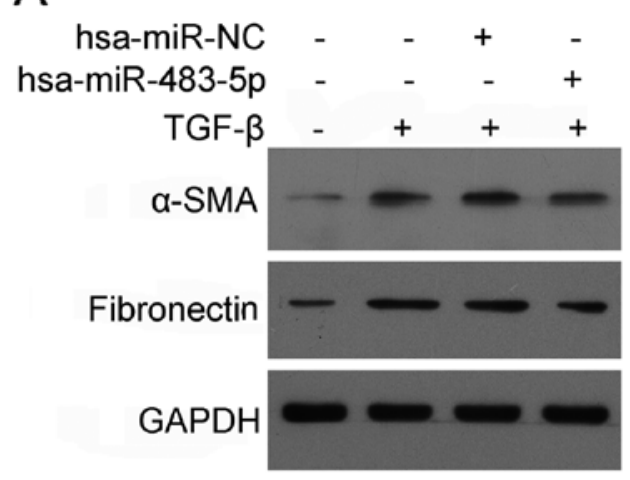

B

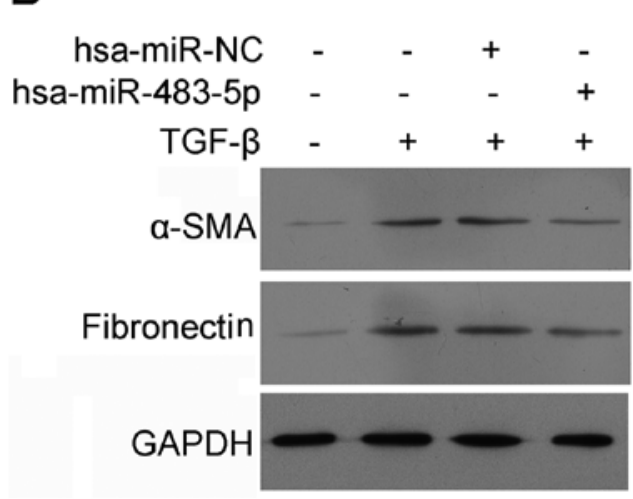

mBEAS-2B

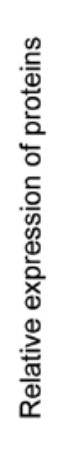

BEAS-2B+TGF- $\beta+N C$

BEAS-2B+TGF- $\beta+$ hsa-miR-483-5p
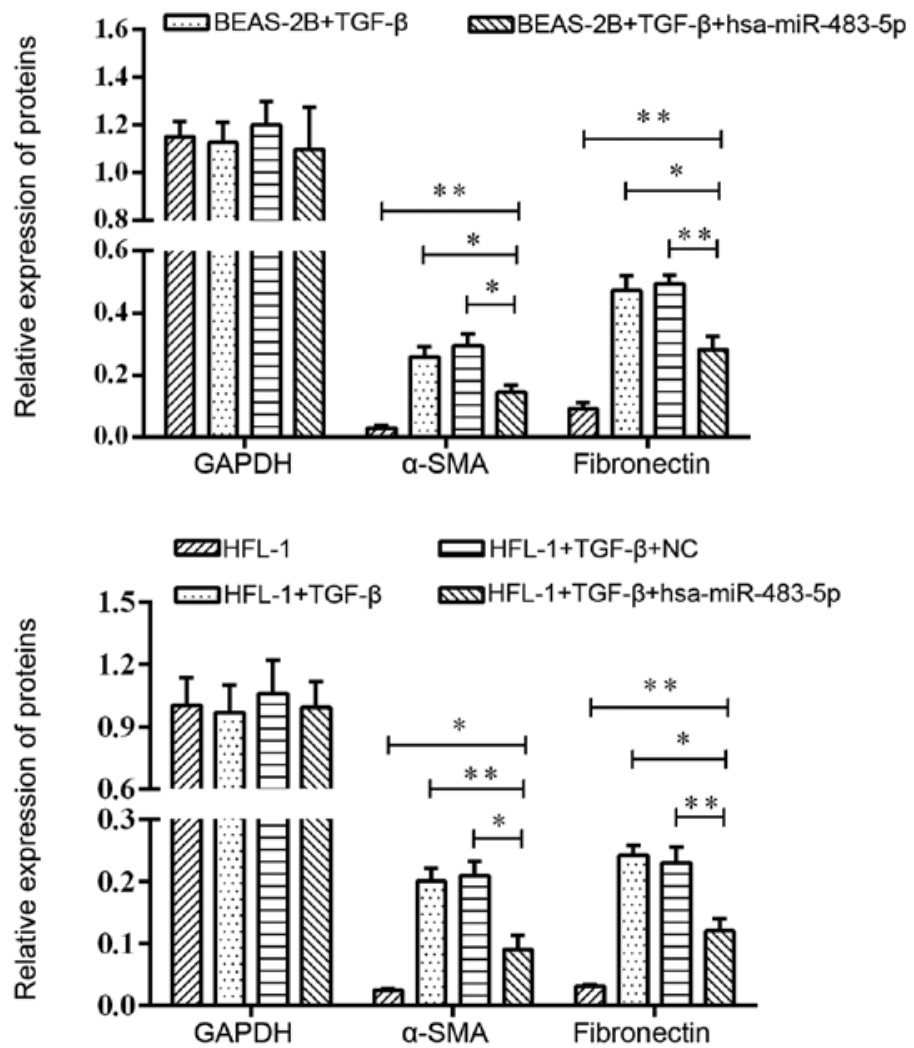

Figure 4. Effect of the overexpression of miR-483-5p on $\alpha$-smooth muscle actin ( $\alpha$-SMA) and fibronectin expression as detected by real-time PCR and western blot analysis in vitro. (A) Protein levels of $\alpha$-SMA and fibronectin in BEAS-2B cells after transforming growth factor- $\beta$ (TGF- $\beta$ ) treatment for $24 \mathrm{~h}$ and miR-483-5p mimics post-transfection for $48 \mathrm{~h}$, compared to the positive control group. (B) Protein levels of $\alpha$-SMA and fibronectin in HFL1 cells after TGF- $\beta$ treatment for $24 \mathrm{~h}$ and miR-483-5p mimics post-transfection for $48 \mathrm{~h}$, compared to the positive control group. All detections were repeated independently for three times. ${ }^{*} \mathrm{P}<0.01$ and ${ }^{* *} \mathrm{P}<0.05$.

from COPD patients when compared with normal smokers by TaqMan low-density array screening. Therefore, in order to clarify the role of miR-483-5p in COPD, miR-483-5p was selected as the target in this study. Based on the results of microarray and RT-PCR, the miR-483-5p expression was significantly decreased ( 2.5-fold reduction) in COPD compared to the healthy controls.
Cigarette smoke or other inhaled irritants activate epithelial cells to release growth factors, such as TGF- $\beta$ and fibroblast growth factor (FGF) which induce fibroblast proliferation, resulting in small-airway inflammation and fibrosis $(28,29)$. Studies have shown that myofibroblasts can be transdifferentiated from fibroblasts in vitro by their exposure to the fibrogenic cytokine TGF- $\beta$ (30-32). Furthermore, others have shown that 
TGF- $\beta$ is a potent stimulus for myofibroblast differentiation and induction of pulmonary fibrosis in vivo $(33,34)$. In addition, Burgess et al (35) used TGF- $\beta$-treated human lung fibroblasts to study pulmonary myofibroblast differentiation. Therefore, in our study, in order to imitate COPD in vitro, TGF- $\beta$ treatment was used to induce a similar condition of COPD in BEAS-2B and HFL1 cells. Our results showed that miR-483-5p transfection significantly abrogated the TGF- $\beta$-mediated decrease in cell proliferation, and $\alpha$-SMA and fibronectin expression in BEAS-2B and HFL1 cells. This indicates that the abrogation of TGF- $\beta$-decreased cell proliferation by miR-483-5p may be associated with the expression of $\alpha$-SMA and fibronectin.

$\alpha$-SMA and fibronectin play important roles during COPD progression. A high molecular weight glycoprotein, fibronectin, is present in the human body as two major isoforms: an insoluble extracellular matrix isomer and a soluble form in the blood (36). The primary function of blood fibronectin is to heal wounds by inducing the reticulo-endothelial system and by mediating cellular adhesion, motility, differentiation, apoptosis and hemostasis (37). This raises the possibility that fibronectin may play an important role in predicting the clinical outcomes in a cohort of patients with mild-to-moderate COPD. Meanwhile, although smooth muscle and endothelial cells also express this marker, $\alpha$-SMA is still the most commonly used but not a specific marker for myofibroblasts (38-41), whose expression is mainly intracellular also in spindle-shaped cells (42). $\alpha$-SMA-positive cells are increased in the airways of COPD patients (43), which suggests that most $\alpha$-SMA-positive cells reveal typical expression profile of myofibroblasts being positive for $\alpha$-SMA, vimentin and negative for desmin (44). Based on our findings, we hypothesis that, during COPD progression, low expression of miR-483-5p may upregulate the expression of these two important proteins by decreasing the chance of binding them directly. In further research, the molecular mechanism through which miR-483-5p regulates $\alpha$-SMA and fibronectin needs to be clarified before miR-483-5p can be developed as a potential molecular biomarker for COPD patients.

In conclusion, we found a new miRNA named miR-483-5p with relatively low expression in COPD patients, which may protect human lung cells by promoting cell growth and activating important proteins such as $\alpha$-SMA and fibronectin. Our findings can provide clues for future functional studies aimed at determining the role of miR-483-5p suppression, as observed in COPD patients, in modulating the adaptive immune balance.

\section{References}

1. Felice KM, Salzman DW, Shubert-Coleman J, Jensen KP and Furneaux HM: The 5' terminal uracil of let-7a is critical for the recruitment of mRNA to Argonaute2. Biochem J 422: 329-341, 2009.

2. Jonas $\mathrm{S}$ and Izaurralde E: Towards a molecular understanding of microRNA-mediated gene silencing. Nat Rev Genet 16: 421-433, 2015.

3. Iorio MV and Croce CM: microRNA involvement in human cancer. Carcinogenesis 33: 1126-1133, 2012.

4. Corvalan AH and Maturana MJ: Recent patents of DNA methylation biomarkers in gastrointestinal oncology. Recent Pat DNA Gene Seq 4: 202-209, 2010.

5. Felicetti F, Errico MC, Segnalini $P$, Mattia $G$ and Carè $A$ : MicroRNA-221 and -222 pathway controls melanoma progression. Expert Rev Anticancer Ther 8: 1759-1765, 2008.
6. Jiang YW and Chen LA: microRNAs as tumor inhibitors, oncogenes, biomarkers for drug efficacy and outcome predictors in lung cancer (Review). Mol Med Rep 5: 890-894, 2012.

7. Reid DJ and Pham NT: Emerging Therapeutic Options for the Management of COPD. Clin Med Insights Circ Respir Pulm Med 7: 7-15, 2013.

8. Lococo F, Cesario A, Del Bufalo A, Ciarrocchi A, Prinzi G, Mina M, Bonassi S and Russo P: Novel therapeutic strategy in the management of COPD: A systems medicine approach. Curr Med Chem 22: 3655-3675, 2015.

9. Wang M, Huang Y, Liang Z, Liu D, Lu Y, Dai Y, Feng G and Wang C: Plasma miRNAs may be promising biomarkers of chronic obstructive pulmonary disease. Clin Respir J 10: 104-111, 2014.

10. Navratilova Z, Kolek V and Petrek M: Matrix metalloproteinases and their inhibitors in chronic obstructive pulmonary disease. Arch Immunol Ther Exp (Warsz) 64: 177-193, 2015.

11. Zanette DL, Rivadavia F, Molfetta GA, Barbuzano FG, Proto-Siqueira R, Silva-Jr WA, Falcão RP and Zago MA: miRNA expression profiles in chronic lymphocytic and acute lymphocytic leukemia. Braz J Med Biol Res 40: 1435-1440, 2007.

12. Xi S, Yang M, Tao Y, Xu H, Shan J, Inchauste S, Zhang M, Mercedes L, Hong JA, Rao M, et al: Cigarette smoke induces C/EBP- $\beta$-mediated activation of miR-31 in normal human respiratory epithelia and lung cancer cells. PLoS One 5: e13764, 2010.

13. Huleihel L, Ben-Yehudah A, Milosevic J, Yu G, Pandit K, Sakamoto K, Yousef H, LeJeune M, Coon TA, Redinger CJ, et al: Let-7d microRNA affects mesenchymal phenotypic properties of lung fibroblasts. Am J Physiol Lung Cell Mol Physiol 306: L534-L542, 2014.

14. Van Pottelberge GR, Mestdagh P, Bracke KR, Thas O, van Durme YM, Joos GF, Vandesompele J and Brusselle GG: MicroRNA expression in induced sputum of smokers and patients with chronic obstructive pulmonary disease. Am J Respir Crit Care Med 183: 898-906, 2011.

15. Mizuno S, Bogaard HJ, Gomez-Arroyo J, Alhussaini A, Kraskauskas D, Cool CD and Voelkel NF: MicroRNA-199a-5p is associated with hypoxia-inducible factor- $1 \alpha$ expression in lungs from patients with COPD. Chest 142: 663-672, 2012

16. Zhu DD, Tang RN, Lv LL, Wen Y, Liu H, Zhang XL, Ma KL and Liu BC: Interleukin-1 $\beta$ mediates high glucose induced phenotypic transition in human aortic endothelial cells. Cardiovasc Diabetol 15: 42, 2016.

17. Liu Y, Pleasants RA, Croft JB, Wheaton AG, Heidari K, Malarcher AM, Ohar JA, Kraft M, Mannino DM and Strange C: Smoking duration, respiratory symptoms, and COPD in adults aged $\geq 45$ years with a smoking history. Int J Chron Obstruct Pulmon Dis 10: 1409-1416, 2015.

18. Johar D, Siragam V, Mahood TH and Keijzer R: New insights into lung development and diseases: The role of microRNAs. Biochem Cell Biol 93: 139-148, 2015.

19. Ma J, Hong L, Xu G, Hao J, Wang R, Guo H, Liu J,Zhang Y, Nie Y and Fan D: miR-483-3p plays an oncogenic role in esophageal squamous cell carcinoma by targeting tumor suppressor EI24. Cell Biol Int 40: 448-455, 2016.

20. Qiao Y, Zhao Y, Liu Y, Ma N, Wang C, Zou J, Liu Z, Zhou Z, Han D, He J, et al: miR-483-3p regulates hyperglycaemiainduced cardiomyocyte apoptosis in transgenic mice. Biochem Biophys Res Commun 477: 541-547, 2016.

21. Wu K, Ma L and Zhu J: miR-483-5p promotes growth, invasion and self-renewal of gastric cancer stem cells by $\mathrm{Wnt} / \beta$-catenin signaling. Mol Med Rep 14: 3421-3428, 2016.

22. Xu H, Yang Y, Zhao H, Yang X, Luo Y, Ren Y, Liu W and Li N: Serum miR-483-5p: A novel diagnostic and prognostic biomarker for patients with oral squamous cell carcinoma. Tumour Biol 37: 447-453, 2016.

23. Qu X, Zhao M, Wu S, Yu W, Xu J, Xu J, Li J and Chen L: Circulating microRNA 483-5p as a novel biomarker for diagnosis survival prediction in multiple myeloma. Med Oncol 31: 219, 2014.

24. Song Q, Xu Y, Yang C, Chen Z, Jia C, Chen J, Zhang Y, Lai P, Fan X, Zhou X, et al: miR-483-5p promotes invasion and metastasis of lung adenocarcinoma by targeting RhoGDI1 and ALCAM. Cancer Res 74: 3031-3042, 2014.

25. Patel D, Boufraqech M, Jain M, Zhang L, He M, Gesuwan K, Gulati N, Nilubol N, Fojo T and Kebebew E: MiR-34a and miR-483-5p are candidate serum biomarkers for adrenocortical tumors. Surgery 154: 1224-1229, 2013.

26. Shen J, Wang A, Wang Q, Gurvich I, Siegel AB, Remotti H and Santella RM: Exploration of genome-wide circulating microRNA in hepatocellular carcinoma: MiR-483-5p as a potential biomarker. Cancer Epidemiol Biomarkers Prev 22: 2364-2373, 2013. 
27. Soeda S, Ohyashiki JH, Ohtsuki K, Umezu T, Setoguchi Y and Ohyashiki K: Clinical relevance of plasma miR-106b levels in patients with chronic obstructive pulmonary disease. Int J Mol Med 31: 533-539, 2013.

28. Retamales I, Elliott WM, Meshi B, Coxson HO, Pare PD, Sciurba FC, Rogers RM, Hayashi S and Hogg JC: Amplification of inflammation in emphysema and its association with latent adenoviral infection. Am J Respir Crit Care Med 164: 469-473, 2001.

29. Barnes PJ: The cytokine network in asthma and chronic obstructive pulmonary disease. J Clin Invest 118: 3546-3556, 2008.

30. Grunstein $\mathbf{M}$ : Histone acetylation in chromatin structure and transcription. Nature 389: 349-352, 1997.

31. Laurent GJ: Regulation of lung collagen production during wound healing. Chest 99 (Suppl 3): 67S-69S, 1991.

32. de Oliveira MV, Silva PL and Rocco PR: Animal models of chronic obstructive pulmonary disease. J Biomedical Sci 5: 1, 2016.

33. Jaiswal AK: Nrf2 signaling in coordinated activation of antioxidant gene expression. Free Radic Biol Med 36: 1199-1207, 2004.

34. Rangasamy T, Cho CY, Thimmulappa RK, Zhen L, Srisuma SS, Kensler TW, Yamamoto M, Petrache I, Tuder RM and Biswal S: Genetic ablation of Nrf2 enhances susceptibility to cigarette smoke-induced emphysema in mice. J Clin Invest 114: 1248-1259, 2004.

35. Burgess HA, Daugherty LE, Thatcher TH, Lakatos HF, Ray DM, Redonnet M, Phipps RP and Sime PJ: PPARgamma agonists inhibit TGF- $\beta$ induced pulmonary myofibroblast differentiation and collagen production: Implications for therapy of lung fibrosis Am J Physiol Lung Cell Mol Physiol 288: L1146-L1153, 2005.

36. Rotundo RF, Vincent PA, McKeown-Longo PJ, Blumenstock FA and Saba TM: Hepatic fibronectin matrix turnover in rats: Involvement of the asialoglycoprotein receptor. Am J Physiol 277: G1189-G1199, 1999.
37. Chou CW, Zhuo YL, Jiang ZY and Liu YW: The hemodynamically-regulated vascular microenvironment promotes migration of the steroidogenic tissue during its interaction with chromaffin cells in the zebrafish embryo. PLoS One 9: e107997, 2014.

38. Ma X, Yang F, Yang S, Rasul A, Li T, Liu L, Kong M, Guo D and Ma T: Number and distribution of myofibroblasts and $\alpha$-smooth muscle actin expression levels in fetal membranes with and without gestational complications. Mol Med Rep 12: 2784-2792, 2015.

39. le Rolle AF, Chiu TK, Fara M, Shia J, Zeng Z, Weiser MR, Paty PB and Chiu VK: The prognostic significance of CXCL1 hypersecretion by human colorectal cancer epithelia and myofibroblasts. J Transl Med 13: 199, 2015.

40. Rossi FW, Napolitano F, Pesapane A, Mascolo M, Staibano S, Matucci-Cerinic M, Guiducci S, Ragno P, di Spigna G, Postiglione L, et al: Upregulation of the $\mathrm{N}$-formyl Peptide receptors in scleroderma fibroblasts fosters the switch to myofibroblasts. J Immunol 194: 5161-5173, 2015.

41. Xiao X, Huang C, Zhao C, Gou X, Senavirathna LK, Hinsdale M, Lloyd P and Liu L: Regulation of myofibroblast differentiation by miR-424 during epithelial-to-mesenchymal transition. Arch Biochem Biophys 566: 49-57, 2015.

42. Dey NB, Foley KF, Lincoln TM and Dostmann WR: Inhibition of cGMP-dependent protein kinase reverses phenotypic modulation of vascular smooth muscle cells. J Cardiovasc Pharmacol 45: 404-413, 2005.

43. Milara J, Serrano A, Peiró T, Gavaldà A, Miralpeix M, Morcillo EJ and Cortijo J: Aclidinium inhibits human lung fibroblast to myofibroblast transition. Thorax 67: 229-237, 2012.

44. Löfdahl M, Kaarteenaho R, Lappi-Blanco E, Tornling G and Sköld MC: Tenascin- $C$ and alpha-smooth muscle actin positive cells are increased in the large airways in patients with COPD. Respir Res 12: 48, 2011. 